\title{
Fully integrated low-loss band-pass filters for wireless applications
}

\author{
M Rais-Zadeh ${ }^{1}$, A Kapoor ${ }^{2}, \mathrm{H}$ M Lavasani ${ }^{2}$ and F Ayazi ${ }^{2}$ \\ ${ }^{1}$ Department of Electrical Engineering and Computer Science, University of Michigan, Ann Arbor, \\ MI 48109, USA \\ ${ }^{2}$ School of Electrical and Computer Engineering, Georgia Institute of Technology, Atlanta, GA 30308, \\ USA \\ E-mail: minar@umich.edu
}

Received 6 February 2009, in final form 27 May 2009

Published 13 July 2009

Online at stacks.iop.org/JMM/19/085009

\begin{abstract}
Fully integrated low insertion loss micromachined band-pass filters are designed and fabricated on the silicon substrate $\left(\rho=10-20 \Omega \mathrm{cm}, \varepsilon_{r}=11.9\right)$ for UHF applications. Filters are made of silver, which has the highest conductivity of all metals, to minimize the ohmic loss. A detailed analysis for realizing low insertion loss and high out-of-band rejection filters using elliptic magnitude characteristics is presented, and a comprehensive model to take into account inductive parasitics of the interconnects is developed. Temperature characteristics of the filters are measured and show stable performance. The presented filters are different from the previously reported lumped element filters in that all filters are fully integrated on silicon substrate and occupy a remarkably smaller die area. Two filters are fabricated using the silver micromachining technique with center frequencies at 1.05 and $1.35 \mathrm{GHz}$. The filters have a constant $3 \mathrm{~dB}$ bandwidth of $300 \mathrm{MHz}$ (28.6\% and 22.2\%) and an insertion loss of 1.4-1.7 dB. The low insertion loss and CMOS compatibility make the presented filters suitable candidates for radio frequency integrated circuits.
\end{abstract}

(Some figures in this article are in colour only in the electronic version)

\section{Introduction}

Micromachined filters have gained interest for advanced integrated RF systems as they offer a high level of integration, low cost and small size. Virtually all low-loss lumped element band-pass filters available today are realized using bulky offchip components or low-loss non-silicon substrates [1, 2]. To date, integration of high-performance lumped element filters on silicon has attracted very little effort, mainly due to the low quality factor $(Q)$ of individual on-chip inductors and capacitors which results in high insertion loss and poor out-of-band rejection in band-pass filters. Over the past few years, thick electroplated copper has been employed in advanced CMOS processes to improve the $Q$ of on-chip passive components, in particular inductors [3, 4]. Although the use of thick copper has a substantial effect on reducing the metal loss, the $Q$ of copper inductors reported in the literature is insufficient for high-order filter applications. Recent research has demonstrated very high-performance silver micromachined passive components with $Q$ s more than
100 that can potentially be integrated with RF circuits $[5,6]$. Such high- $Q$ and CMOS-compatible passives are highly desirable for realization of low-loss lumped element band-pass filters and high-performance matching networks in a small form-factor on a single silicon chip, as they do not require additional board area for interconnection and packaging.

Previously, we reported on the implementation of micromachined lumped element band-pass filters with insertion loss in the range of $2-5 \mathrm{~dB}$ on silicon [7]. In this work, we elaborate on the design, fabrication strategies and modeling of the filters to improve the frequency response and demonstrate two low-loss $(<1.7 \mathrm{~dB})$ band-pass filters in the UHF range. We explain the physical and practical limits of achieving the required filter performance and identify the $Q$ of individual passive components. We determine the temperature characteristics of the lumped silver filters by measuring the frequency response of the filters at elevated temperatures. The lumped filters presented in this work can find several applications in communication circuits. In this 
Table 1. Range of component values that can be effectively integrated with planar lumped filters.

\begin{tabular}{llll}
\hline & Minimum & Maximum & Maximum $Q$ \\
\hline Inductance & $0.5 \mathrm{nH}$ & $10 \mathrm{nH}$ & $>80$ \\
Capacitance & $0.1 \mathrm{pF}$ & $10 \mathrm{pF}$ & $>300$ \\
\hline
\end{tabular}

paper, we demonstrate the application of these lumped filters in attenuating the spurious and harmonic modes of high- $Q$ thickness-mode piezo-acoustic filters.

The micromachined band-pass filters presented in this paper exhibit a low insertion loss on CMOS-grade silicon substrates $(\rho=10-20 \Omega \mathrm{cm})$. Moreover, these filters present a good out-of-band rejection of more than $22 \mathrm{~dB}$ while occupying less than $8.8 \mathrm{~mm}^{2}$ of the die area. To our knowledge, these integrated lumped filters represent state-ofthe-art performance on CMOS-grade silicon at this frequency range.

\section{Design procedure}

Maximally flat [8], coupled resonator, Chebychev [2, 9, 10], and elliptic filter configurations [11] are extensively used in RF systems. For a given order filter, among these commonly used filter topologies, the magnitude characteristic of elliptic filters drops off more steeply than others, providing the sharpest cutoff [12]. In addition, values of the lumped components used in elliptic filters can be selected in a range that are realizable using surface micromachining techniques (table 1), allowing effective integration of MEMS components with planar structures. This not only simplifies the fabrication of such filters, but also minimizes the die area. A drawback to elliptic filters is their nonlinear phase delay, in which its importance has to be determined based on the filter application. The application of lumped filters in this work is selecting closely located channels in the UHF range, which calls for spurious-free filters exhibiting a sharp cutoff and a high outof-band rejection. To achieve this, lumped element filters are interfaced with narrow-band channel-select acoustic filters to attenuate their spurious modes and enhance their out-ofband rejection. Elliptic topology is the best candidate for this application.

The magnitude characteristic of an elliptic filter offers equal ripples in both the passband and the stopband. However, in an integrated implementation, neither the value nor the $Q$ of lumped components exactly matches the initial design value. This is due to fabrication imperfections and parasitics, resulting in a quasi-elliptic response for the filter. For this reason, the measured response of an elliptic filter might resemble that of a quasi-elliptic filter.

In this paper, we considered the following specifications for the elliptic filters: passband ripple of $1 \mathrm{~dB}$, minimum outof-band attenuation of $25 \mathrm{~dB}$ at an offset frequency of $300 \mathrm{MHz}$ and fixed 3-dB bandwidth of $300 \mathrm{MHz}$. Since elliptic filters have a sharp cutoff, the $1 \mathrm{~dB}$ bandwidth of filters is designed to be close to the $3 \mathrm{~dB}$ bandwidth. The $3 \mathrm{~dB}$ bandwidth of filters is adjusted when optimizing the physical layout of the filters using electromagnetic simulations, as discussed in section 3 .

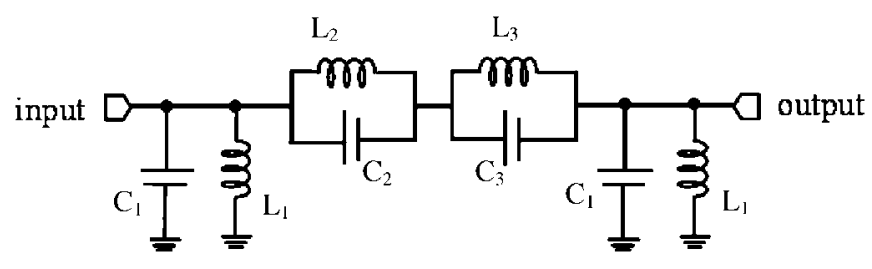

Figure 1. Circuit diagram for a third-order band-pass elliptic filter.

Table 2. Normalized values for the realization shown in figure 1 .

\begin{tabular}{lllllll}
\hline$f_{0}(\mathrm{GHz})$ & $L_{1}(\mathrm{H})$ & $L_{2}(\mathrm{H})$ & $L_{3}(\mathrm{H})$ & $C_{1}(\mathrm{~F})$ & $C_{2}(\mathrm{~F})$ & $C_{3}(\mathrm{~F})$ \\
\hline 1.05 & 0.168 & 0.226 & 0.363 & 5.91 & 2.76 & 4.42 \\
1.35 & 0.131 & 0.187 & 0.271 & 7.60 & 3.68 & 5.35 \\
\hline
\end{tabular}

To demonstrate the versatility of the fabrication process, two filters are designed at $1.05 \mathrm{GHz}$ and $1.35 \mathrm{GHz}$.

It is determined that a third-order filter satisfies the attenuation specifications for both filters. After determining the order, the normalized low-pass network configuration with $1 \Omega$ termination and $1 \mathrm{~dB}$ ripple is obtained using established tables for double-resistance-terminated ladder elliptic filters [12]. The low-pass configuration is then transformed to the normalized band-pass configuration shown in figure 1 [13]. This configuration utilizes two parallel-tuned circuits where each condition of parallel resonance directly corresponds to a transmission zero. Normalized element values for this configuration can be found in table 2. As shown in table 2, the component values are extended in a reasonable range $(\approx 2)$.

The final step is to scale the normalized band-pass filter to the required center frequency $\left(\Omega_{0}\right)$ and termination impedance $(Z)$. Such a conversion is performed via

$$
C=\frac{C_{n}}{\Omega_{0} \times Z} \quad \text { and } \quad L=\frac{L_{n}}{\Omega_{0}} \times Z .
$$

From equation (1), it is evident that the component values of a filter with larger termination impedances are more practical (i.e. smaller $C$ and larger $L$ ). Consequently, the termination impedance of the filters in this work is increased to $150 \Omega$ from the standard $50 \Omega$ so that capacitances decrease to offer higher $Q \mathrm{~s}$ and inductances increase to reach a practical value. Besides the fact that $150 \Omega$ offers suitable component values, it is the optimum termination impedance for the proceeding acoustic filters. Component values for the topology shown in figure 1 with $150 \Omega$ terminations are listed in table 3 .

Initial simulations and design modifications are performed in the Agilent ADS using lumped element models for inductors and capacitors. In simulations, $Q$ s of the inductors and capacitors at the center frequency of each filter are taken as 60 and 120, respectively. The simulated magnitude response of the filters, when terminated to $150 \Omega$, is shown in figure 2 . The respective insertion loss and return loss are $1.5 \mathrm{~dB}(1.7 \mathrm{~dB})$ and $7 \mathrm{~dB}(8 \mathrm{~dB})$ for the filter at $1.05 \mathrm{GHz}(1.35 \mathrm{GHz})$. The group delay of the filter is determined using the expression suggested by Shapir [14] and is shown in figure 3. The simulated group delay at the midband is $\sim 2 \mathrm{~ns}$ and the maximum variation of 
Table 3. Component values for the integrated elliptic band-pass filters.

\begin{tabular}{lllllll}
\hline$f_{0}(\mathrm{GHz})$ & $L_{1}(\mathrm{nH})$ & $L_{2}(\mathrm{nH})$ & $L_{3}(\mathrm{nH})$ & $C_{1}(\mathrm{pF})$ & $C_{2}(\mathrm{pF})$ & $C_{3}(\mathrm{pF})$ \\
\hline 1.05 & 3.82 & 5.14 & 8.25 & 5.97 & 2.79 & 4.47 \\
1.35 & 2.32 & 3.30 & 4.79 & 5.97 & 2.89 & 4.20 \\
\hline
\end{tabular}

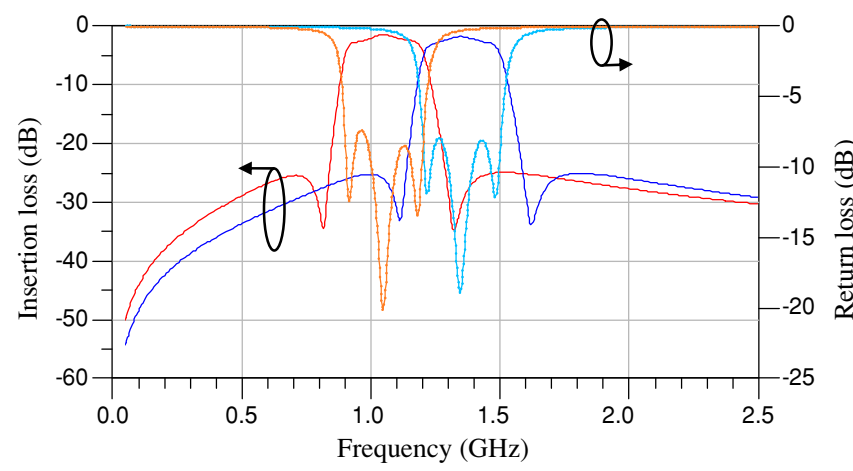

Figure 2. Agilent ADS simulated magnitude response of band-pass elliptic filters.

the group delay in the passband is within $3.8 \mathrm{~ns}$, as shown in figure 3 .

\section{Layout optimization}

Optimizations on the physical layout of the filters are performed in the Sonnet 2.5D EM simulation tool [15]. Sonnet simulations are performed using the Sonnet thick metal model with two sheets. The physical layout of the band-pass filters is finalized by optimizing the layout of the incorporated passives to get the desired frequency response for each individual LC tank. The frequency of the transmission zeros is adjusted such that a $3 \mathrm{~dB}$ bandwidth of $300 \mathrm{MHz}$ is obtained for both filters. The length of the lines interconnecting the tanks is maintained as short as possible to minimize parasitic effects. The Sonnet simulated response of the filters is shown in figure 4. To account for the surface roughness and impurities in the electroplated film, the silver conductivity is taken as $5.8 \times$ $10^{7} \mathrm{~S} \mathrm{~m}^{-1}$, which is smaller than the text value for the bulk conductivity $\left(6.3 \times 10^{7} \mathrm{~S} \mathrm{~m}^{-1}\right)$ [16]. The optimized EM simulated insertion loss and return loss of the filter at $1.05 \mathrm{GHz}(1.35 \mathrm{GHz})$ are $1.3 \mathrm{~dB}(1.7 \mathrm{~dB})$ and $7.5 \mathrm{~dB}(7 \mathrm{~dB})$, respectively.

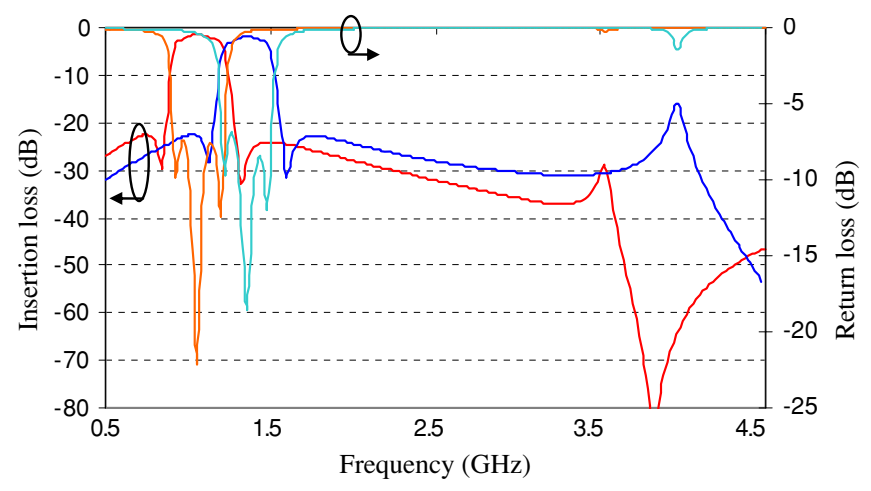

Figure 4. EM simulation response of the third-order band-pass elliptic filters.

\section{Fabrication technique}

Filters are fabricated using the CMOS-compatible process introduced in [5]. The schematic view of the fabrication process is shown in figure 5. The substrate is first passivated with a dielectric layer to reduce the parasitic of the pads and to physically support the devices at the final step. A $4 \mu \mathrm{m}$ thick gold is evaporated and patterned to define the routing layer. Next, a $1 \mu \mathrm{m}$ thick plasma-enhanced chemical vapor-deposited (PECVD) silicon dioxide layer is deposited at $300{ }^{\circ} \mathrm{C}$ and patterned. A $20 \mu \mathrm{m}$ thick silver layer is then electroplated into a photoresist mold. The mold and the seedlayer are subsequently removed. Finally, the loss of the silicon substrate is eliminated by selective etching of the silicon underneath the filters from the backside, leaving behind a firmly supported dielectric membrane. It is worth mentioning that, the filters can be encapsulated at the wafer level using the process outlined in [5]. Filters are fabricated on a $3 \mu \mathrm{m}$ thick silicon dioxide membrane. We chose silicon dioxide as the membrane material because it is widely available in CMOS processes. Round symmetrical inductors are employed in the filter as they exhibit higher $Q$ than the conventional spiraltype inductors. Figures 6 and 7 show SEM views of the
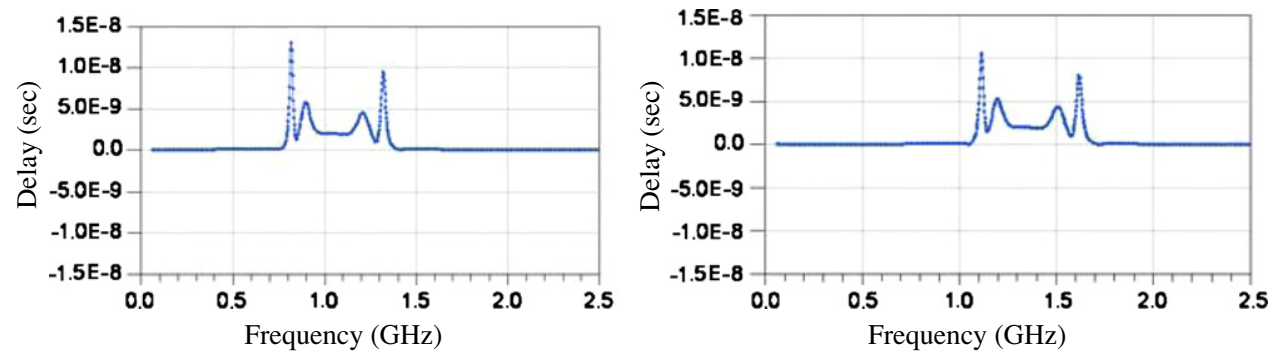

Figure 3. Group delay calculated using the Shapir's formula corresponding to (left) filter at $1.05 \mathrm{GHz}$ and (right) filter at $1.35 \mathrm{GHz}$. 


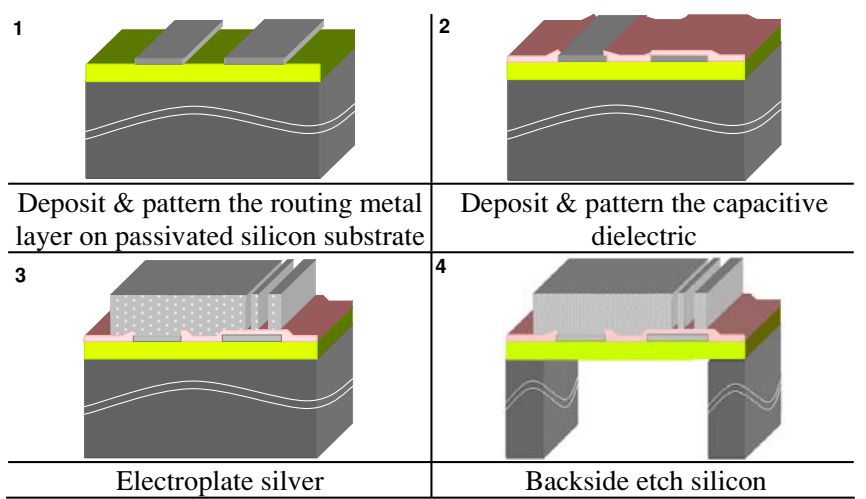

Figure 5. Fabrication process of integrated silver band-pass filters.

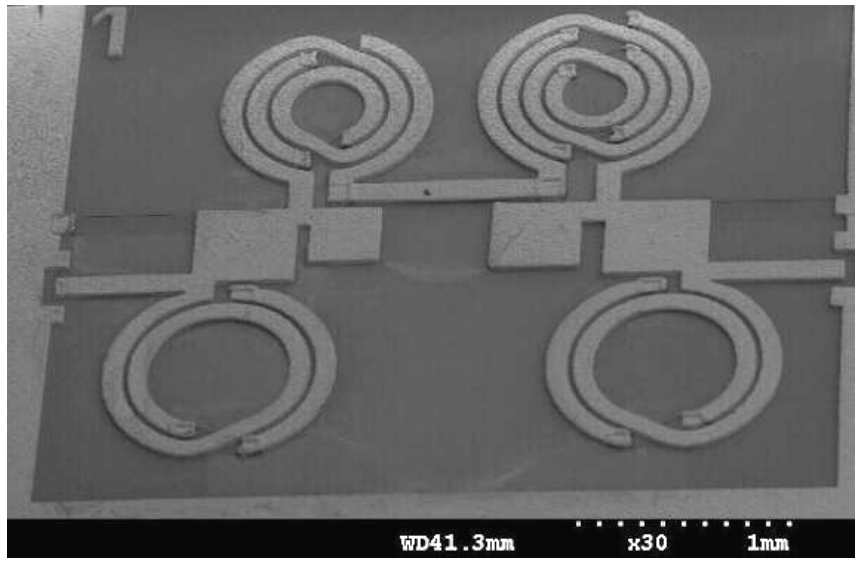

Figure 6. SEM of the $1.05 \mathrm{GHz}$ filter fabricated on the $3 \mu \mathrm{m}$ oxide membrane, showing that the filter occupies $2.5 \mathrm{~mm} \times 3.5 \mathrm{~mm}$ of the die area.

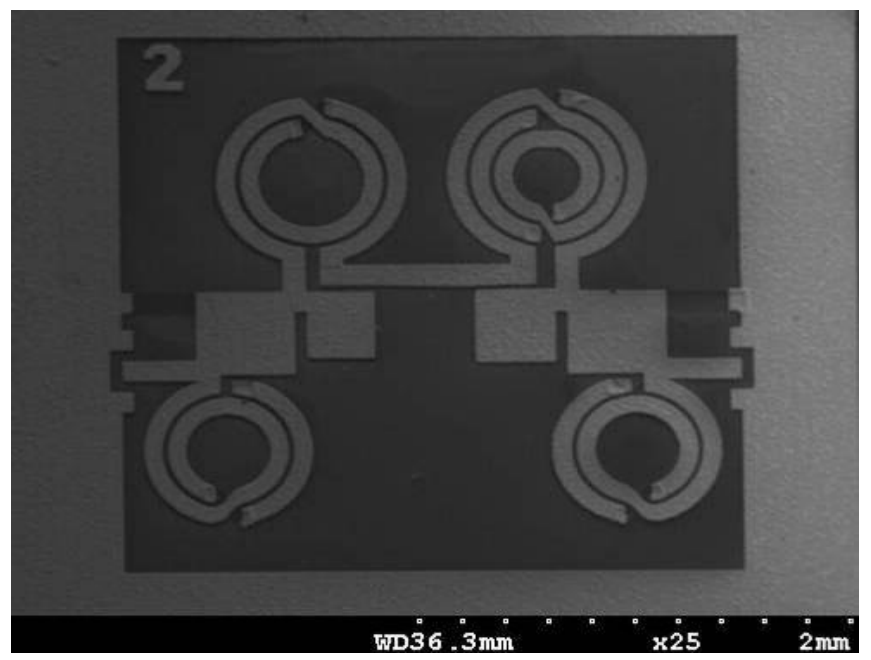

Figure 7. SEM of the $1.35 \mathrm{GHz}$ filter fabricated on the $3 \mu \mathrm{m}$ silicon dioxide membrane, showing that the filter occupies $2.1 \mathrm{~mm} \times$ $2.4 \mathrm{~mm}$ of the die area.

fabricated filters on the silicon dioxide membrane. As shown, with a capacitive gap of $1 \mu \mathrm{m}$, each filter occupies less than $8.8 \mathrm{~mm}^{2}$.

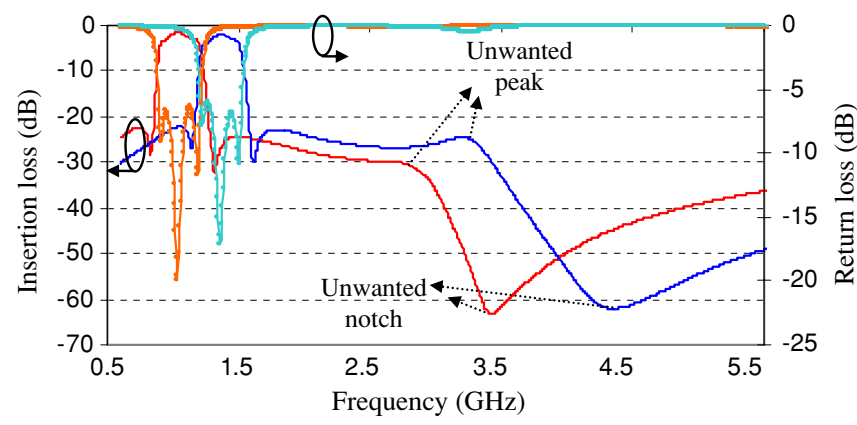

Figure 8. Measured frequency response of silver band-pass filters fabricated on the silicon dioxide membrane.

\section{Results and discussion}

On-wafer S-parameter measurements of the fabricated bandpass filters and individual lumped components are carried out using an Agilent E8364B PNA and Cascade GSG infinity (I-50) microprobes. Accurate measurement of component $Q$ s in excess of 100 requires a very thorough calibration. Calibration is performed using a SOLT calibration procedure on Cascade $50 \Omega$ impedance standard substrate (101-190). Pad parasitics are not de-embedded from the filter frequency response to avoid underestimation of the filter loss. The filter response when terminated to $150 \Omega$ is then measured by converting the termination impedance of the PNA to $150 \Omega$. To ensure repeatability in the measurements, devices are measured several times, and each time the calibration is redone.

\subsection{Measured response}

Figures 8 and 9 show the measured frequency response of the silver band-pass filters, when terminated to $150 \Omega$. The measured bandwidth of the filters is very close to the design value, namely $296 \mathrm{MHz}$ at $1.052 \mathrm{GHz}$ and $290 \mathrm{MHz}$ at $1.368 \mathrm{GHz}$. The measured insertion loss at the center frequency of the $1.05 \mathrm{GHz}(1.35 \mathrm{GHz})$ filter is $1.4 \mathrm{~dB}(1.7 \mathrm{~dB})$. The out-of-band rejection of both filters is better than $22 \mathrm{~dB}$ in a broad frequency range. The low insertion loss and high out-of-band rejection of these filters make them suitable candidates for wireless applications. As discussed earlier, due to fabrication imperfections, the tanks do not exhibit equal $Q$ s; hence, the passband ripples are not equal and the response of the filters is quasi-elliptic. The extracted group delay of the filters is shown in figure 10. As shown in figure 10, the measured delay response is in good agreement with the simulated response shown in figure 3 .

\subsection{Measured versus modeled}

To investigate the source of unwanted resonances shown in figure 8, filters are modeled in the ADS. Figure 11 shows the electrical model of a band-pass elliptic filter on the silicon dioxide membrane. The interconnect lines shown in figures 6 and 7 are modeled as an inductor and a small series resistor (not shown in figure 11). It is found that the parasitic of these lines is the dominant source of the unwanted resonances. More 


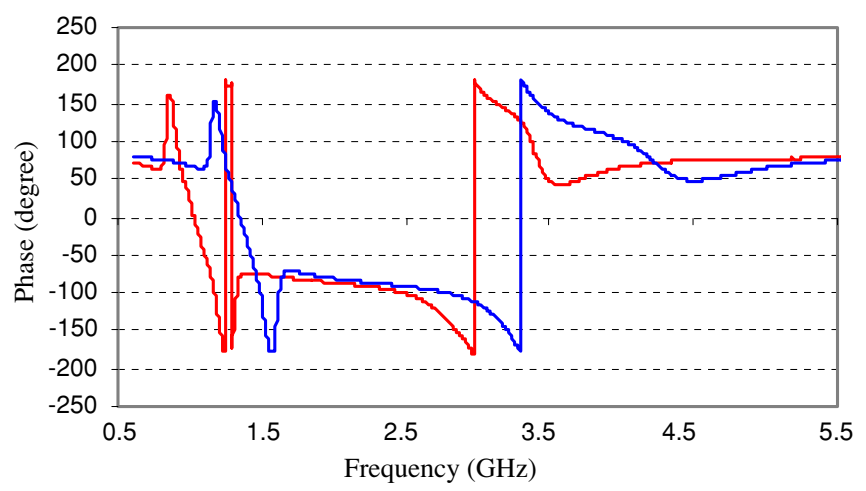

Figure 9. Measured phase response of the band-pass filters.

accurately, $L_{\mathrm{p} 1}, L_{\mathrm{p} 1}^{\prime}, L_{\mathrm{p} 11}$, and $L_{\mathrm{p} 11}^{\prime}$ define the unwanted high frequency notch while $L_{\mathrm{p} 2}, L_{\mathrm{p} 3}$, and $L_{\mathrm{p} 23}$ define the unwanted high frequency peak. There is a small coupling inductance between the closely located planar spiral inductors. However, in the UHF range, these mutual inductances are small and their effect can be ignored. Figures 12 and 13 compare the measured response of the filters with the modeled response. As shown, the measured and modeled responses are in excellent agreement. The minimum $Q$ of inductors and capacitors at the center frequency of each filter is obtained from the fitted electrical model to be 50 and 110, respectively.

\subsection{Temperature characteristic}

The temperature stability of the filters is tested in a temperature-controlled RF probe station from Desert Cryogenics. In this section, results of the filter at $1.35 \mathrm{GHz}$ are reported. Similar behavior is observed for the filter at $1.05 \mathrm{GHz}$. To study the effect of removing the silicon on the temperature stability of integrated silver filters, the response of two identical filters is measured, where silicon is not removed from backside of one filter (the other filter is on a silicon dioxide membrane). Over the range of $25-80{ }^{\circ} \mathrm{C}$, the filter on the silicon dioxide membrane shows a much stable performance compared to the filter on the passivated silicon substrate (figure 14). This is due to the large capacitive parasitics of the silicon substrate, which show significant temperature dependence. Table 4 compares the maximum drift

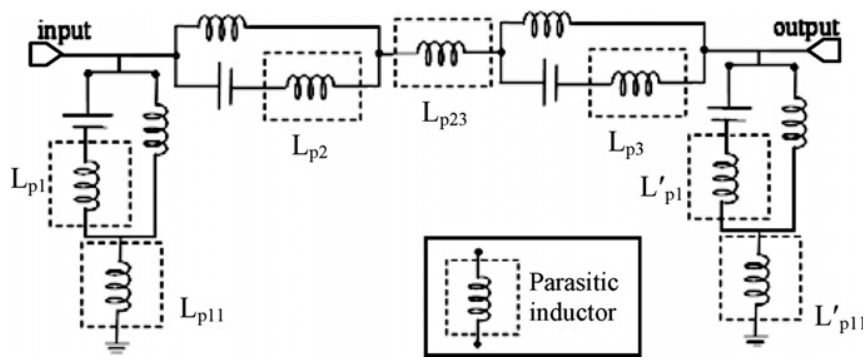

Figure 11. Electrical model of elliptic silver filters on the silicon dioxide membrane. The parasitic of interconnects is modeled as low- $Q$ inductors.

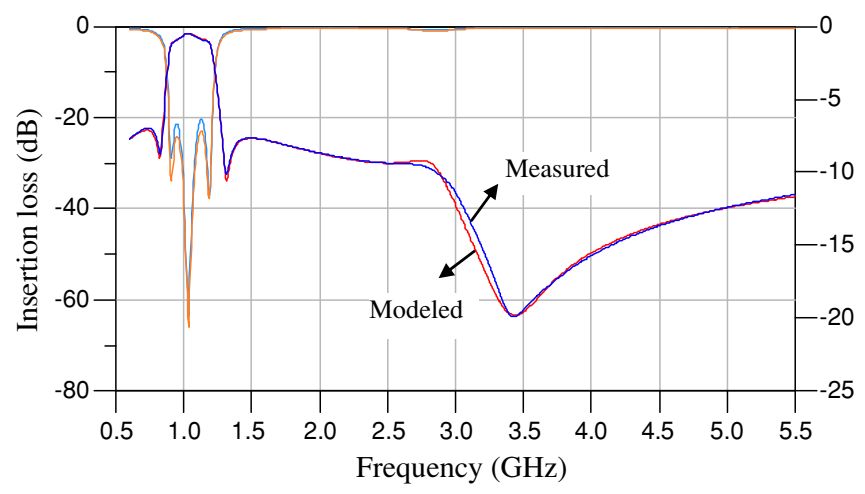

Figure 12. Measured and modeled frequency response of the filter at $1.05 \mathrm{GHz}$ using the electrical model shown in figure 11 .

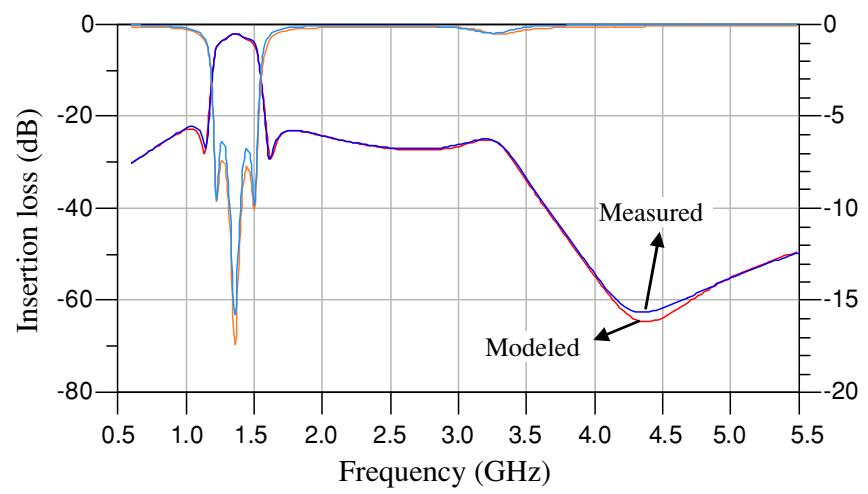

Figure 13. Measured and modeled frequency response of the $1.35 \mathrm{GHz}$ filter using the electrical model shown in figure 11.

of the frequency response of the filters. It should be noted that the temperature test took several hours resulting in a slight

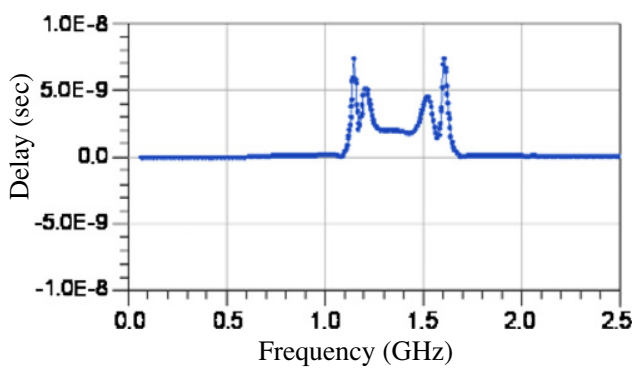

Figure 10. Extracted group delay calculated using the Shapir's formula for the (left) filter at $1.05 \mathrm{GHz}$ and (right) filter at $1.35 \mathrm{GHz}$. 
Table 4. Comparison of the drift in the frequency response of filters on silicon and when the silicon is etched from the backside, showing that the temperature stability is improved by removing the silicon.

\begin{tabular}{llll}
\hline Substrate & Center frequency drift (\%) & $3 \mathrm{~dB}$ bandwidth drift (\%) & Insertion loss drift (\%) \\
\hline Silicon & 0.264 & 0.320 & 8.33 \\
Silicon dioxide membrane & 0.036 & 0.411 & 4.74 \\
\hline
\end{tabular}

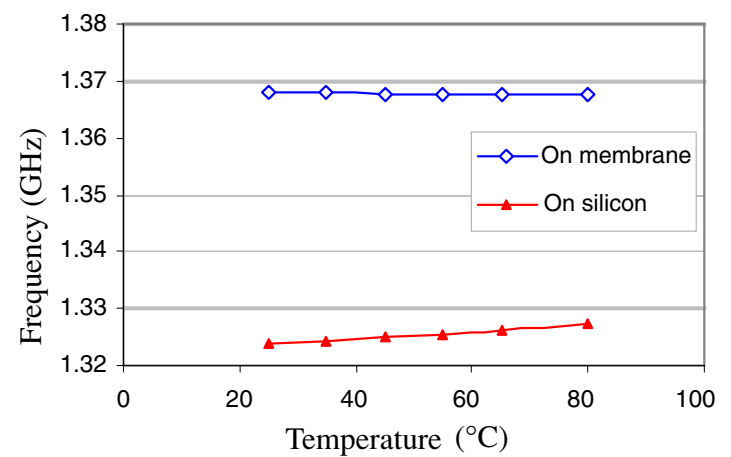

(a)

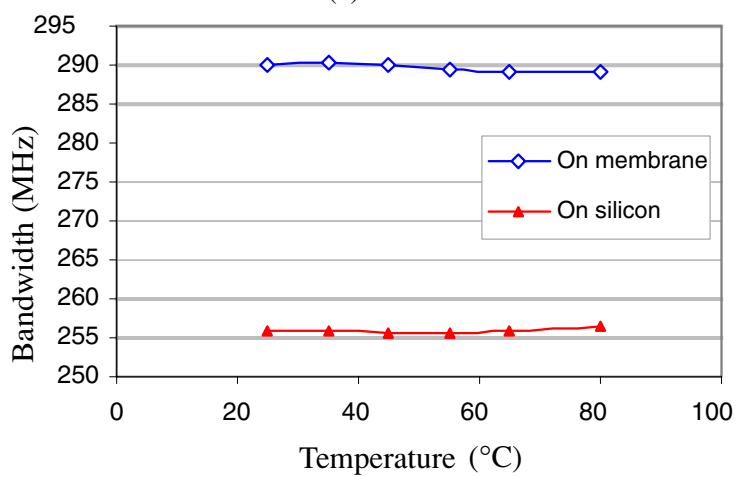

(b)

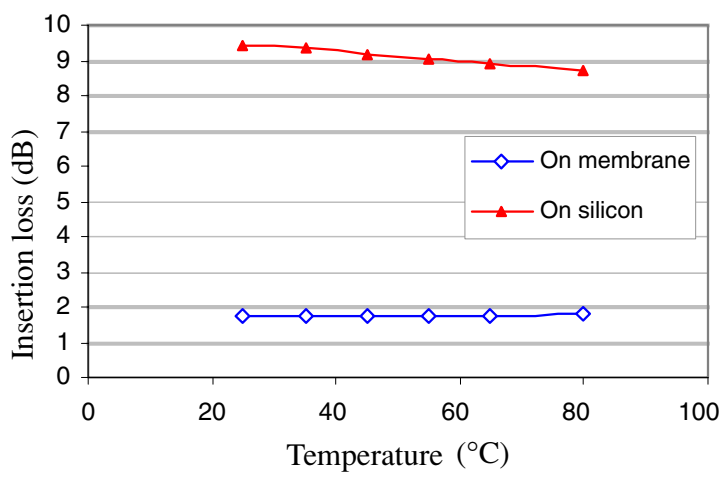

(c)

Figure 14. (a) Frequency, $(b) 3-\mathrm{dB}$ bandwidth, and $(c)$ insertion loss behavior of the $1.35 \mathrm{GHz}$ filter (on the CMOS-grade silicon and silicon dioxide membrane) in the $25-80{ }^{\circ} \mathrm{C}$ temperature range.

degradation in the calibration that might be a contributing factor in the accuracy of the measurements.

\subsection{Individual component $Q$}

The $Q$ of the filter components is measured on individual test structures. The characteristics of an exemplary inductor and capacitor incorporated in the filters are shown in figures 15 and 16. The pad-only characteristics are measured on the open

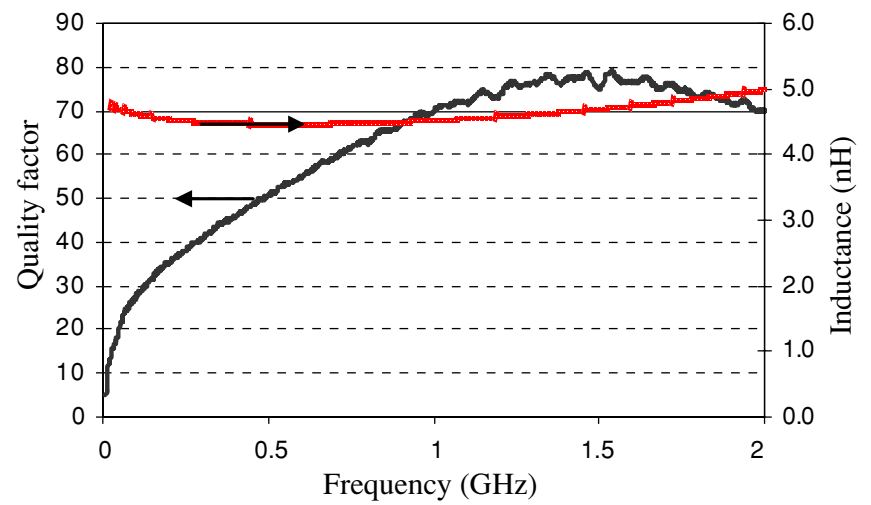

Figure 15. De-embedded characteristic of a silver inductor on a silicon dioxide membrane.

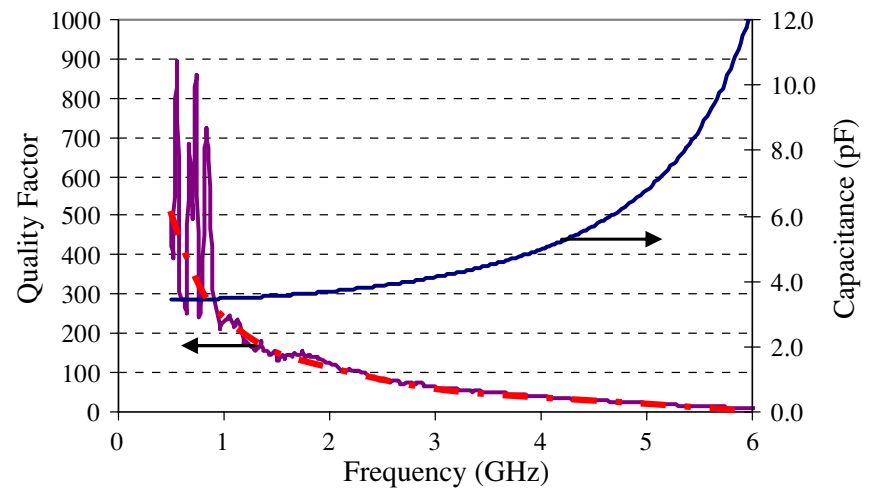

Figure 16. De-embedded characteristic of a silver capacitor on a silicon dioxide membrane. The dashed red line represents the trade line of the $Q$.

pad structures. The pads parasitics are then de-embedded from the overall component characteristic by subtracting the Y-parameters of the pads from the Y-parameters of the embedded inductors and capacitors. As shown, lumped silver components exhibit a very high $Q$ on the silicon dioxide membrane. The fluctuation in the $Q$ is due to the high accuracy required for measuring $Q$ in excess of 100 which is not possible using on-wafer S-parameter measurement.

\subsection{Interfacing with acoustic filters}

To demonstrate the application of fabricated lumped filters in improving the response of small-bandwidth acoustic filters, the filter at $1.35 \mathrm{GHz}$ is cascaded with a high- $Q$ acoustic filter at $1.49 \mathrm{GHz}$. The piezo-electrically transduced acoustic filter is fabricated on a $2 \mu \mathrm{m}$ SOI substrate as reported by Pan et al [17]. The response of the acoustic filter is shown in figure 17. As shown, the high-order acoustic filter exhibits 


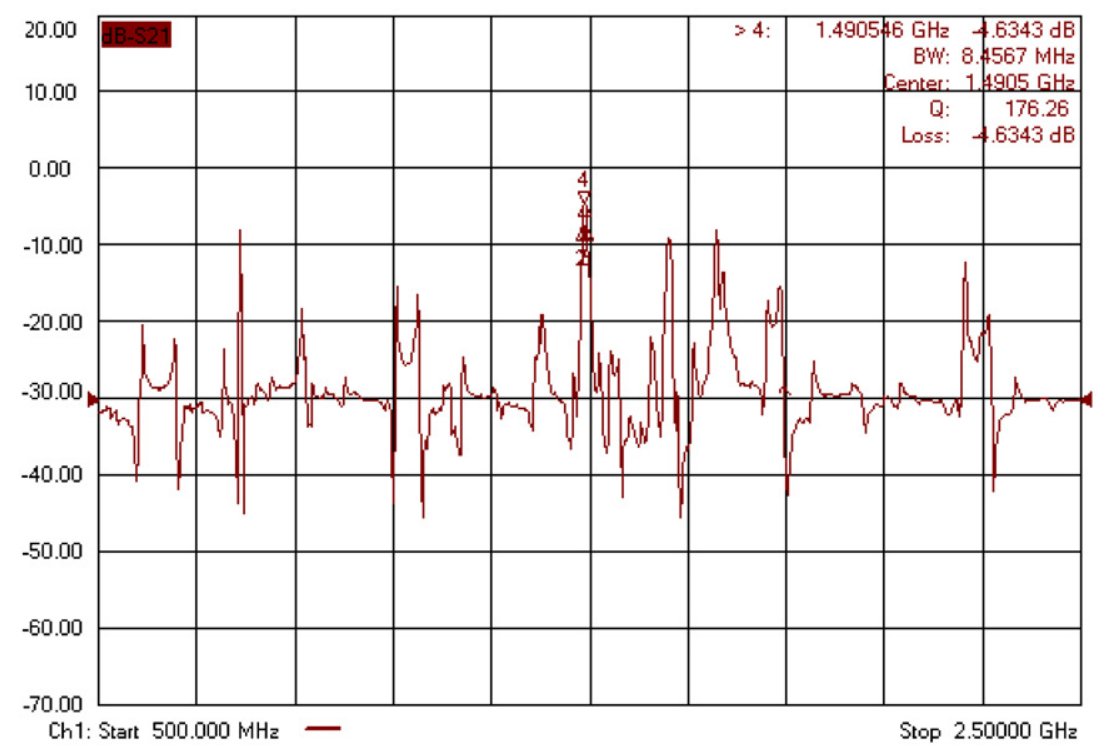

Figure 17. Measured response of an exemplary high-order acoustic filter at $1.49 \mathrm{GHz}$, showing an insertion loss of $4.6 \mathrm{~dB}$ and bandwidth of $8.4 \mathrm{MHz}$.

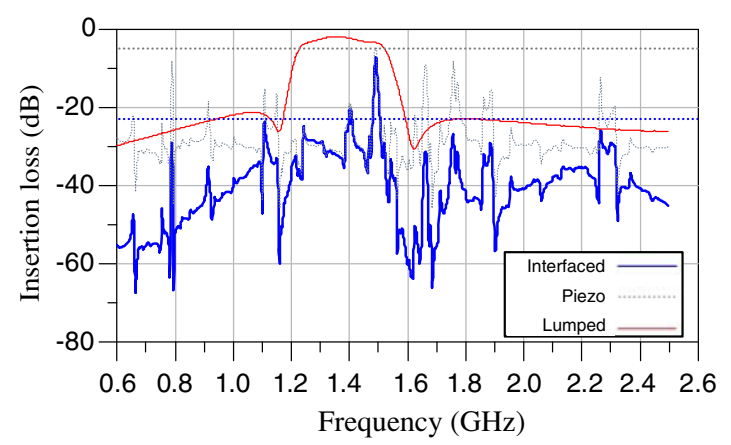

Figure 18. Comparison of the frequency response of a high-order acoustic filter before and after cascading with the lumped band-pass filter, showing that the lumped filter suppresses the spurious modes by more than $18 \mathrm{~dB}$.

several harmonic and spurious resonances with an attenuation of less than $5 \mathrm{dBc}$ from the desired mode at $1.49 \mathrm{GHz}$. The elliptic lumped filter would greatly attenuate these modes without introducing significant loss.

As a proof of concept, the measured response of the lumped filter at $1.35 \mathrm{GHz}$ is cascaded with that of the acoustic filter. The S-parameters of the lumped elliptic band-pass filter as well as the acoustic filter are imported into the ADS design as data items. The effect of the bondwire connecting the two filters is modeled as an inductor in series with a small resistor. Assuming a single $3 \mathrm{~mm}$ long aluminum bondwire with a diameter of $1.5 \mathrm{mil}$, the effective inductance and resistance of the bondwire are calculated from the expressions given in [18] to be $3 \mathrm{nH}$ and $0.47 \Omega$, respectively. Figure 18 shows the response of the cascaded lumped and acoustic filters. As expected, the spurious modes of the cascaded filters are damped by more than $23 \mathrm{dBc}$, an improvement of $18 \mathrm{~dB}$ over the stand-alone acoustic filter. The piezo-acoustic filter is not perfectly matched to $150 \Omega$. The lumped filter provides matching between the $150 \Omega$ termination and acoustic filter.
Therefore, as shown, insertion loss of the cascaded filter array is less than the sum of the loss of the lumped filter and the acoustic filter. The center frequency of the acoustic filter is close to the $3 \mathrm{~dB}$ frequency of the lumped filter (figure 18), resulting in a higher insertion loss for the cascaded filters compared to the case where the center frequencies are overlapping. In conclusion, the lumped filter greatly improves the performance of the acoustic filter without introducing a significant loss.

\section{Conclusion}

Design and characterization of integrated band-pass filters implemented on the CMOS-grade silicon substrate were reported. Band-pass filters were demonstrated in the UHF range with measured insertion loss of less than $1.7 \mathrm{~dB}$. The low insertion loss, CMOS-compatibility and stable performance over temperature of the presented filters make them strong candidates for RF integrated circuits.

\section{Acknowledgment}

This work was supported by Defense Advanced Research Projects Agency (DARPA) under Analog Spectral Processors project. Authors would like to thank Dr Wanling Pan for fabricating the acoustic filter and the staff at Georgia Tech Microelectronic Research Center for their assistance.

\section{References}

[1] Borwick R L, Stupar P A, DeNatale J F, Anderson R and Erlandson R 2003 Variable MEMS capacitors implemented into RF filter systems IEEE Trans. Microw. Theory Tech. 51 315-9

[2] Entesari K, Obeidat K, Brown A R and Rebeiz G M 2007 A 25-75-MHz RF MEMS tunable filter IEEE Trans. Microw. Theory Tech. 55 2399-405 
[3] Yoon J B, Choi Y S, Kim B and Yoon E 2002

CMOS-compatible surface-micromachined suspended-spiral inductors for multi-GHz silicon RF ICs IEEE Electron Device Lett. 23 591-3

[4] Raieszadeh M, Yoon S W, Monajemi P, Laskar J and Ayazi F High-Q integrated inductors on trenched Si islands Proc. IEEE Micro Electro Mechanical Systems Conf. (MEMS 2005) (Miami, FL, January 2005) pp 199-202

[5] Rais-Zadeh M, Kohl P A and Ayazi F High-Q micromachined silver passives and filters Tech. Dig. IEEE Int. Electron Device Meeting (IEDM '06) (San Francisco, CA, December 2006) pp 727-30

[6] Carazzeti P, Dubois M A and de Rooij N F High performance micromachined RF planar inductors IEEE Int. Conf. on Solid-State Sensors, Actuators and Microsystems (Transducers '05) (Seoul, Korea, June 2005) pp 1084-7

[7] Rais-Zadeh M, Lavasani H M and Ayazi F CMOS-compatible encapsulated silver band-pass filters IEEE Int. Microwave Symp. (IMS '07) (Honolulu, Hawaii, June 2007) pp 1301-4

[8] Chen R T, Brown E R and Bang C A A compact low-loss Ka-band filter using 3-dimensional micromachined integrated coax Proc. IEEE Int. Conf. on Microelectromechanical Systems (Maastricht, Netherlands, January 2004) pp 801-4

[9] Lugo C, Thompson D and Papapolymerou J 2003

Reconfigurable band-pass filter with variable bandwidth at $5.8 \mathrm{GHz}$ using a capacitive gap variation technique $33 \mathrm{rd}$ European Microwave Conf. (Munich, October 2003) vol 3 pp 923-6
[10] Palego C, Pothier A, Crunteanu A, Chatras M, Blondy P, Champeaux C, Tristant P and Catherinot A 2008 A two-pole lumped-element programmable filter with MEMS pseudodigital capacitor banks IEEE Trans. Microw. Theory Tech. 56 729-935

[11] Ching G S Design and practical implementation of a $2.4 \mathrm{GHz}$ lumped element elliptic band-pass filter Proc. Asia-Pacific Microwave Conf. (Taiwan, December 2001) vol 2 pp 523-6

[12] Huelsman L P 1993 Active and Passive Analog Filter Design, An Introduction (New York: McGraw-Hill)

[13] Williams A B and Taylor F J 1988 Electronic Filter Design Handbook-LC, Active, and Digital Filters 2nd edn (New York: McGraw-Hill)

[14] Shapir I Suggestions for a new formula to calculate group-delay from frequency domain measurements Proc. 36th European Microwave Conf. (Manchester, UK, September 2006) pp 1233-6

[15] www.sonnetusa.com

[16] Chan J E, Sivaprasad K and Chamberlin K A 2007 High-frequency modeling of frequency-dependent dielectric and conductor losses in transmission lines IEEE Trans. Compon. Packag. Technol. 30 86-91

[17] Pan W, Abdolvand R and Ayazi F A low-loss $1.8 \mathrm{GHz}$ monolithic thin-film piezoelectric-on-substrate filter IEEE Int. Conf. on Microelectromechanical Systems (MEMS'08) (Tuscan, AZ, January 2008) pp 1032-5

[18] Hoang J and Lee R Microwave wirebond model for die to laminate 2004 HFSS Users Workshop (Los Angeles, CA, February 2004) 